\title{
Where is the machine looking? Locating discriminative light-scattering features by class-activation mapping
}

\author{
Patricio Piedra ${ }^{a}$, Christian Gobert ${ }^{\mathrm{a}}$, Aimable Kalume ${ }^{a}$, Yong-Le Pan ${ }^{\mathrm{a}}$, Miroslav Kocifaj ${ }^{\mathrm{b}, \mathrm{c}}$, \\ Karri Muinonen ${ }^{\mathrm{d}, \mathrm{e}}$, Antti Penttilä ${ }^{\mathrm{d}}$, Evgenij Zubko ${ }^{\mathrm{f}}$, Gorden Videen ${ }^{\mathrm{a}, \mathrm{f}, \mathrm{g}, *}$ \\ a U.S. Army Research Laboratory, 2800 Powder Mill Road, Adelphi, MD, USA \\ b ICA, Slovak Academy of Sciences, Dúbravská Road 9, Bratislava 845 03, Slovak Republic

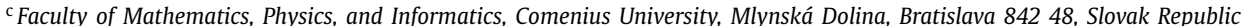 \\ ${ }^{\mathrm{d}}$ Department of Physics, University of Helsinki, P.O. box 64, FI 00014, Finland \\ e Geospatial Research Institute FGI, National Land Survey, Geodeetinrinne 2, Masala, FI 02430, Finland \\ ${ }^{\mathrm{f}}$ Humanitas College, Kyung Hee University, 1732, Deogyeong-daero, Giheung-gu, Yongin-si, Gyeonggi-do 17104, South Korea \\ ${ }^{\mathrm{g}}$ Space Science Institute, 4750 Walnut Street, Boulder Suite 205, CO 80301, USA
}

\section{A R T I C L E I N F O}

\section{Article history:}

Received 3 October 2019

Revised 7 February 2020

Accepted 1 March 2020

Available online 2 March 2020

\section{Keywords:}

Light scattering

Deep learning

\begin{abstract}
A B S T R A C T
We explore a technique called class-activation mapping (CAM) to investigate how a Machine Learning (ML) architecture learns to classify particles based on their light-scattering signals. We release our code, and also find that different regions of the light-scattering signals play different roles in ML classification. These regions depend on the type of particles being classified and on the nature of the data obtained and trained. For instance, the Mueller-matrix elements $S_{11}^{*}, S_{12}^{*}$ and $S_{21}^{*}$ had the greatest classification activation in the diffraction region. Linear polarization elements $S_{12}^{*}$ and $S_{21}^{*}$ were most accurate in the backscattering region for clusters of spheres and spores, and was most accurate in the diffraction region for other particle classes. The CAM technique was able to highlight light-scattering angles that maximize the potential for discrimination of similar particle classes. Such information is useful for designing detector systems to classify particles where limited space or resources are available, including flow cytometry and satellite remote sensing.
\end{abstract}

(c) 2020 The Authors. Published by Elsevier Ltd. This is an open access article under the CC BY-NC-ND license. (http://creativecommons.org/licenses/by-nc-nd/4.0/)

\section{Introduction}

Optical characterization of particles is an important application of light scattering. Elastic scattering has the largest cross-section compared to the inelastic scattering processes [1], the components for an elastic light-scattering detector system are relatively inexpensive, such a system can be automated, and analyses can be made without collecting samples. While particles can be analyzed using Raman or fluorescence spectra [2-4], such analyses are performed on spectra that are typically orders of magnitude less intense than those in elastic scattering. While some recent devices have been constructed to analyze aerosols in situ, aerosol samples are often collected because the inelastic light-scattering signals are so weak. The light sources are typically in the UV for fluorescence excitation, costing significantly more than visible sources.

\footnotetext{
* Corresponding author.

E-mail address: gorden.w.videen.civ@mail.mil (G. Videen).
}

As a result, there has been significant effort dedicated to finding discriminating patterns among elastic light-scattering data [5-10]. Such discriminating features could include, for instance, the phase function, dynamical time-resolved analysis [39], or degree of polarization [40].

In recent years, ML [11] has proven to be useful in finding accurate decision-boundary limits, which allow for very fine discrimination of particle shapes from light-scattering information. For instance, Kaye et al. [12] studied the performance of radial-basisfunction neural networks to detect hazardous respirable fibers from light scattering. These results were limited to only three classes of particles and only a limited portion of the forward scattering was used. Misclassifications were around 20\%. However, modern architectures of neural networks such as convolutional neural networks or successive subspace learning [13] have demonstrated accurate results for classifying 2D and 3D datasets. The results of implementing convolutional neural networks for shape classification of complex-shaped particles using elastic lightscattering data was explored by us previously [14]. In that study, 
we found that convolutional neural networks (CNN) yield accurate classification of the shape of particles whose scattering intensity or degree-of-polarization distribution was collected at $1^{\circ}$ resolution. Our ML architecture was able to classify complex shapes from highly variant light-scattering data inputs. However, a major drawback of ML is that it is difficult for humans to comprehend how the intermingling of numerous tensor weights and biases of deep learning eventually lead to these decision boundaries that yield discrimination. Nonetheless, in these weights and biases lie important clues regarding decision-making characteristics about the dataset. If we can open the so-called ML black box, we can mitigate the non-intuitive nature of ML and furthermore leverage ML as an avenue to promote discovery of discriminative features. In this study, we focus on understanding how ML yields discrimination between different classes of light-scattering datasets and try to bring some physical intuition regarding ML classification. We apply a technique called class-activation mapping $[15,16]$ to examine which part of the light-scattering phase function has the most influence in making the classification. Such information is valuable for the experimentalist to know where to put a detector to get the best results. The CAM tells, in effect, where the ML algorithm looks to find discriminating features among the different classes of particles. In addition, we describe and release our code for others to use on their datasets. Our code, which we named ScatCAM, can be accessed at: https://github.com/USArmyResearchLab/ScatCAM/ .

\section{Methods}

This study uses the dataset that was synthetically generated by Piedra et al. [14], which consists of seven classes of particles with the following characteristics: complex shape, rotated stochastically and isotropically, of refractive index $1.5+\mathrm{i} 0$, and size parameter 5 . We kept the size and refractive index constant for all model particles in this study in order to focus on the dependence of particle morphology. For instance, we are quite aware that transparent soot particles, represented by pre-fractal clusters of spheres, do not exist in the visible spectral region, but we also know that the inclusion of a realistic absorbing component would introduce features attributable to the absorbing component, that would further complicate the analyses. In addition, we also restrict the size parameter, so this factor does not play a role in the classification. This latter restriction may seem quite limiting for practical applications, but in actuality, there are many methods that can be used to determine aerosol size, e.g., $[17,18]$. Such techniques even can be incorporated into the instrumentation used to capture the lightscattering patterns. When we consider that the sizing can be determined using such a parallel technique, then this restriction is not so limiting. For the purposes of this discussion, we briefly discuss some foundations for ML to provide an understanding of neural networks followed by a description of the network architecture used in this investigation. We specifically address the creation of a class-activation map (CAM) through the use of a global-average pooling (GAP) layer. Lastly, we discuss the selection of hyperparameters and the training environment.

\subsection{Convolutional neural networks}

CNNs are multi-stage algorithms that learn features with trainable convolutional filters for classification. CNNs outperform artificial neural networks for high-resolution image classification through the use of the convolutional operator [19-21]. Convolutional processing is usually paired with pooling, which downsamples neighboring pixels into a single pixel, thereby increasing the scope of a convolutional filter once a feature map or image has been pooled. A CNN usually contains a processing sequence of
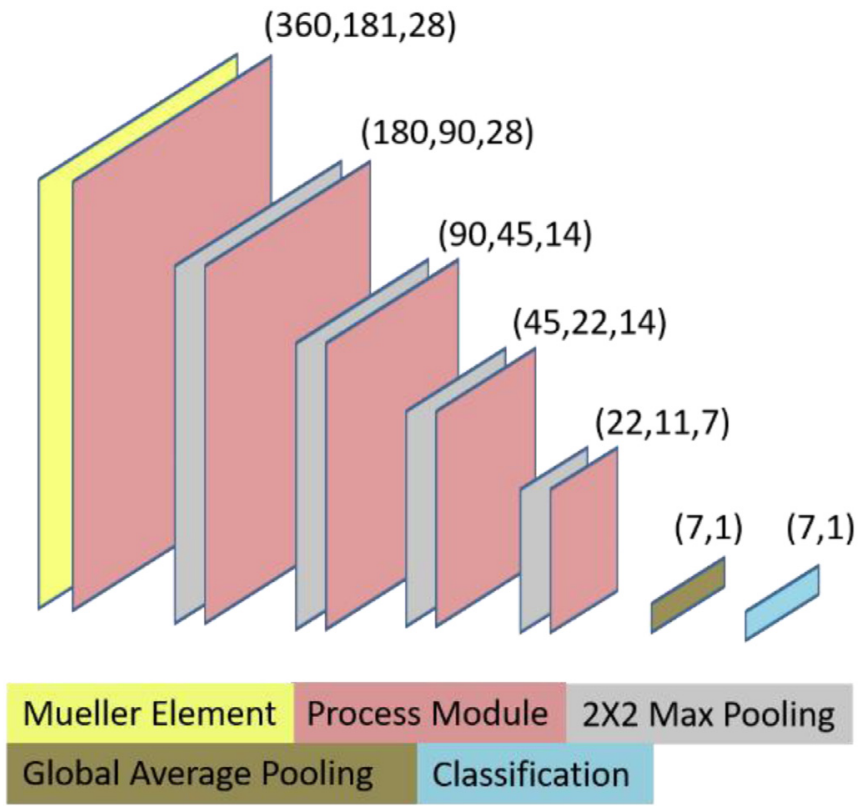

Fig. 1. Convolutional neural network designed for this study. The quantities in parentheses indicate the dimensions of our data processing. The global-average pooling layer [15] was originally invented as a regularization layer, but it also allows for class-activation mapping [16].

convolutional filters and pooling layers to down-sample the original input into a feature space that can be handled by a set of fully connected neurons for classification. Neural networks gain their non-linear discrimination ability from the use of activation functions at each node or filter output. A loss function calculates the error between input and prediction. Learnable parameters are updated with stochastic gradient descent to minimize the loss function. Hyperparameters are those excluded from the learning and are chosen before network training.

\subsection{Network architecture}

Essentially, a digital image is a $\Theta \times \Phi \times P$ matrix of information, where $\Theta$ is the number of pixels in the vertical direction, $\Phi$ is the number of pixels in the horizontal direction, and $P$ is the number of colors (i.e., typically red, blue and green). In a lightscattering dataset, $\Theta$ is the number of datapoints of the polar angle, $\Phi$ is the number of datapoints in the azimuthal angle, and $P$ is the scattering information input (e.g., scattering intensity, degree of polarization, degree of circular polarization, etc.). One could also incorporate wavelength dependence.

We use Python and the Keras library [22] to implement the ML tool. The network used in this investigation is comprised of multiple processing modules that are connected sequentially. $2 \times 2$ maximum pooling, down-sampling by taking the maximum value in a $2 \times 2$ pixel region, is performed in between the connections of processing modules. After four sequences of process modules and pooling a final process module, a GAP layer and classification layer is attached to complete the network. A schematic of the network architecture used is displayed in Fig. 1.

Each process module contains $3 \times 3$ convolutional operators of stride 1, leaky ReLU activation with alpha of 0.3 [23], batch normalization [24], and Res-net inspired residual connections [25]. The architecture within a single process module is depicted in Fig. 2.

Batch normalization mitigates internal covariant shift, maintaining the distribution of features so as to speed up learning [24]. Residual connections reduce the effect of diminishing gradient dur- 


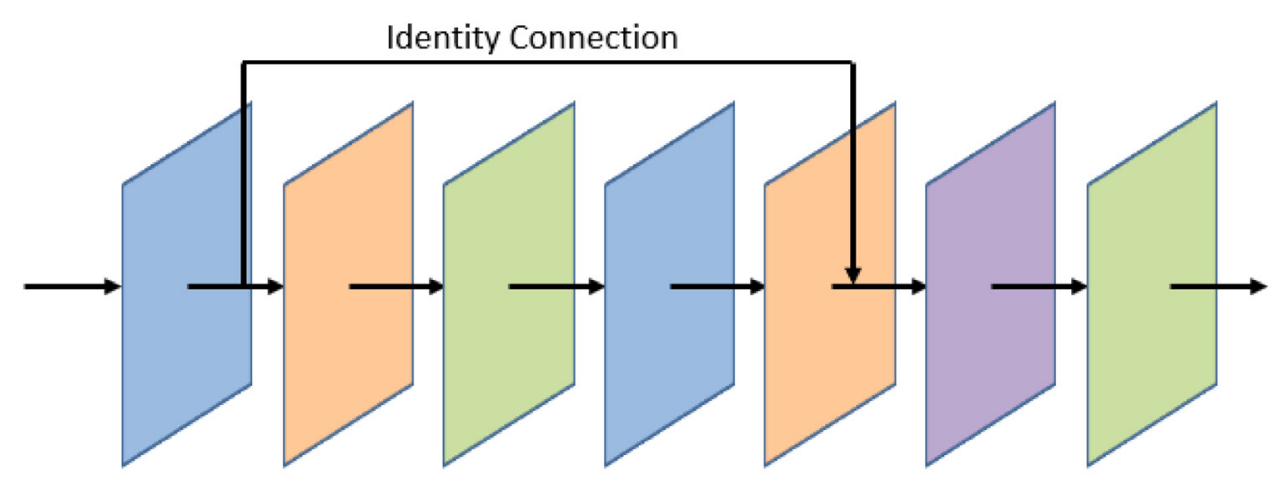

3×3 Convolution Batch Normalization Leaky ReLU Feature Map Summation

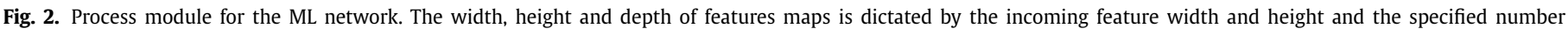
of filters of the process module respectively.

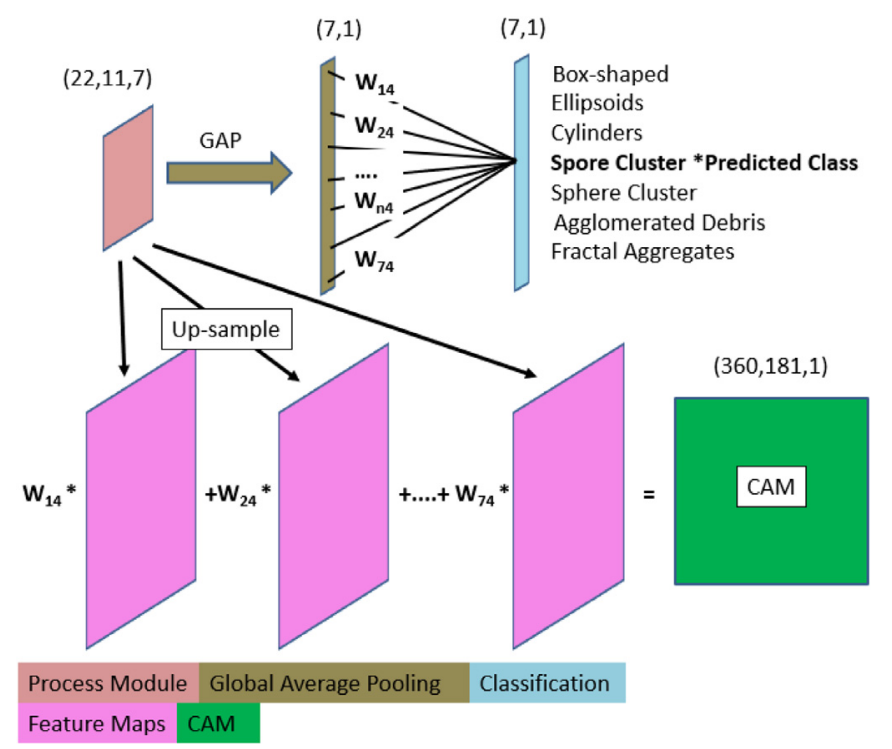

Fig. 3. Creating a class-activation map (CAM) for an instance of spore-cluster prediction. The feature maps of the final convolutional layer are up-sampled to the original input size and multiplied by the corresponding classification weights. The CAM is assembled from the summation of the feature maps.

ing backpropagation and encourage the learning of residual information [25]. Leaky ReLU activations provide non-saturating activations with defined gradients [23]. The final layers include the global average pooling layer across the final 7 features maps connected to 7 neurons with softmax [23] activation for classification.

\subsection{Class-activation maps with gap layers}

CAMs are generated from the interaction between the final convolutional layer, GAP layer and classification layer. A CAM indicates the highest activated regions used by the CNN for classification [16].

Each feature map of the last convolutional layer is up-sampled to the original input height and width through bilinear interpolation. Each up-sampled feature map is then multiplied by the corresponding weight of the predicted class connection between the GAP and classification layer. The features maps are then summed together along the feature map axis. A diagram of the CAM generation for an input Mueller matrix and predicted class is depicted in Fig. 3.

\subsection{Network training environment}

Three networks are trained for each Mueller matrix element $\bar{S}=\left[S_{i j}(\theta, \phi)\right] ; i, j=1,2,3,4$, where $\theta$ is the polar angle and $\varphi$ is the azimuthal angle. We partition each $\bar{S}=\left[S_{i j}(\theta, \phi)\right]$ data set into three subsets for 3-fold cross-validation experimental scheme. For each of the three folds, two subsets are used for training and one subset is used for validation. The loss function is cross-entropy with respect to the class shapes. L2 regularization [26] is used for filter weights, with a value 0.01 . Optimization is performed with stochastic gradient descent using a learning rate scheduler [27]. The initial learning rate is 0.001 and reduce incrementally by a factor of 0.2 to a minimum learning rate of 0.00001 . We stop network training when the loss function no longer decreases after 12 iterations or a maximum of 100 epochs is reached. An epoch is defined as a set of training iterations with the number of iterations equal to training set size divided by the batch size. Each batch is randomly sampled from the training set. Batch size was set to 25 . Loss and accuracy are recorded during network training across the 3-fold cross-validated schemes.

\section{Results}

\subsection{An example to illustrate our results}

For the purposes of this discussion, let us briefly discuss one example that will demonstrate how we analyzed our dataset. The light-scattering Mueller matrix is a $4 \times 4$ transformation of the initial light-beam Stokes vector to the 4-dimensional Stokes vector $\vec{I}=[I, Q, U, V]^{T}$ as described by [28-30]. The Mueller matrix describes the electromagnetic state of the scattered light and is a function of the polar angle $\theta$ and azimuthal angle $\varphi$; mathematically, $\bar{S}=\left[S_{i j}(\theta, \phi)\right]$; for $i, j=1,2,3,4$. Each of the elements of the Mueller matrix is an inherent property of the light-scattering system and describes a transformation of the incident Stokes vector $\vec{I}$ to a scattered Stokes vector $\vec{I}_{s c a}$; i.e., $\vec{I}_{s c a}=S \vec{I}$. For example, Fig. 4 illustrates two elements of the light-scattering Mueller matrix from a spore cluster, one of the seven groups discussed by Piedra et al. [14].

The light-scattering total intensity distribution $S_{11}$ acts as the magnitude of the transformation $\bar{S}$. In experiments, it is difficult to measure the absolute magnitude and the element is usually normalized at some scattering angle, e.g. $\theta=10^{\circ}$. We are interested in highlighting the importance of two-dimensional structure in the distribution of light scattering. Hence, in our training set, we normalized $S_{11}$ such that scattering in the forward direction 

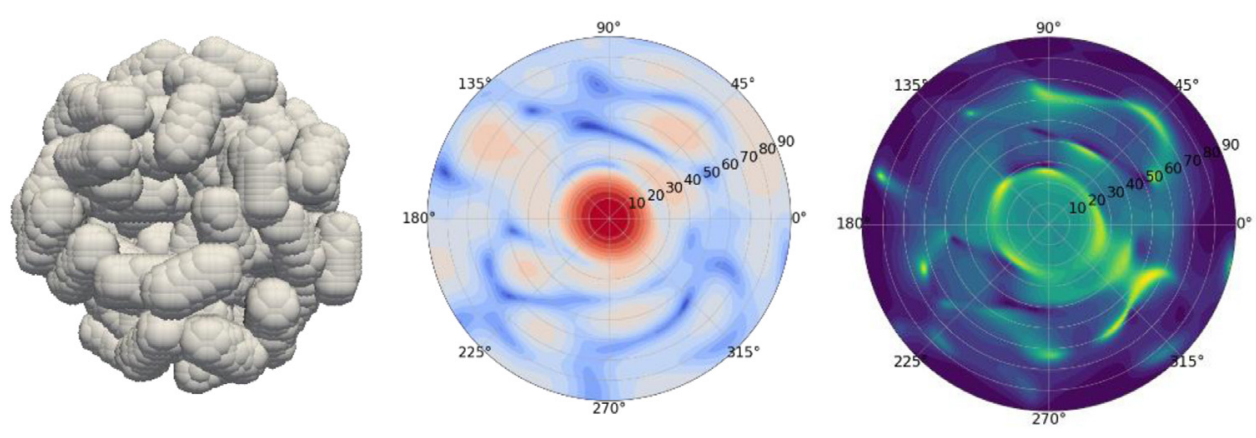

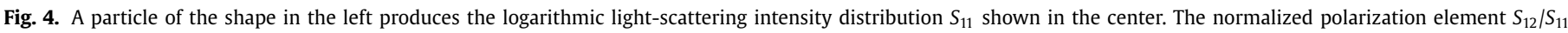

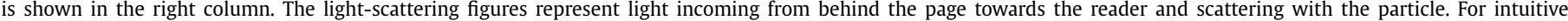
clarity, we show only the forward hemisphere scattering from $0^{\circ} \leq \theta \leq 90^{\circ}$ and $0^{\circ} \leq \varphi \leq 360^{\circ}$.

Table 1

Maximum and minimum values of recall, precision and F1 score for the $S_{12}^{*}$ 3-fold cross-validation experiments .

\begin{tabular}{llll}
\hline Class & F1_Score & Precision & Recall \\
\hline BOX & $(1,1)$ & $(1,0.99)$ & $\mathbf{( 0 . 9 9 , 0 . 9 9 )}$ \\
CYLINDER & $(1,0.99)$ & $(1,0.99)$ & $\mathbf{( 1 , 0 . 9 9 )}$ \\
ELLIPSOID & $(1,0.96)$ & $(1,0.96)$ & $(\mathbf{1 , 0 . 9 7 )}$ \\
SPHERES & $(0.96,0.95)$ & $(0.96,0.94)$ & $\mathbf{( 0 . 9 7 , 0 . 9 6 )}$ \\
SPORES & $(0.97,0.95)$ & $(0.97,0.96)$ & $\mathbf{( 0 . 9 6 , 0 . 9 4 )}$ \\
DUST & $(1,0.99)$ & $(1,0.99)$ & $\mathbf{( 0 . 9 9 , 0 . 9 9 )}$ \\
FRACTAL & $(0.99,0.99)$ & $(0.99,0.99)$ & $\mathbf{( 0 . 9 9 , 0 . 9 9 )}$ \\
\hline
\end{tabular}

$\theta=0^{\circ}$ is 1 . In this manner, the ML architecture cannot simply distinguish scattering particles by differences in the intensity of the forward direction, and we force the ML to learn patterns of the light-scattering distribution along $\theta$ and $\varphi$. Furthermore, for the training set of all other elements of $\bar{S}$ other than $S_{11}$, all quantities have been normalized with respect to $S_{11}$; i.e., $S_{i j} / S_{11}$ for $S_{i j} \neq$ $S_{11}$. For the purposes of brevity, henceforth in our discussion, when we refer to $S_{11}^{*}$, we are referring to a normalized $S_{11}$ with respect to forward scattering, while $S_{i j}^{*} \neq S_{11}^{*}$ is referring to the polarization matrix elements normalized to the total intensity:

$S_{i j}^{*}=\frac{S_{i j}}{S_{11}}$, for $S_{i j} \neq S_{11}$

\subsection{Network training results}

Across the 16 Mueller matrix components, we performed 3-fold cross-validation experiments, and 48 networks were trained. We display a portion of training diagnostics from a single 3 -fold crossvalidation experiment of the $S_{12}^{*}$ Mueller matrix component to highlight the network convergence. Similar results were observed across all other Mueller components and the same analysis was performed for each.

Recall, precision and F1 score are described by Fawcett [31]. Recall represents the correct classification of a class. Precision represents the number of correct classification within the predicted classes. F1 score is the harmonic mean of the recall and precision. We present the validation subset maximum and minimum recall, precision, and F1 score for classification across the 3-fold crossvalidation experiments for each class in Table 1 . These are values between 0 and 1 , where large values indicate better performance.

The agreement of asymptotic loss (Fig. 6) and accuracy (Fig. 5) across the cross-validation experiments provides evidence for network convergence and lack of over-fitting. Similar results were observed across the cross-validation experiments for other Mueller-

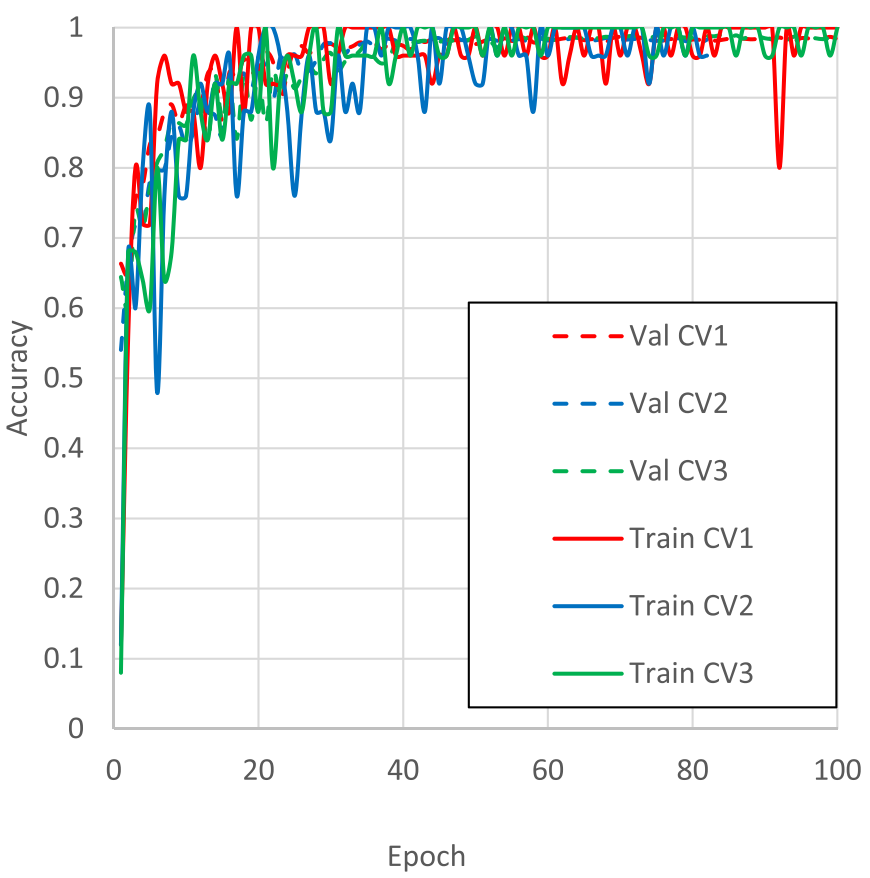

Fig. 5. Plot of accuracy over each epoch in training for the validation and training subsets during cross-validation experiments.

matrix components. The incorporation of data processing layers (i.e., Fig. 2) to our CNN architecture yielded significant improvements in ML performance compared to the results previously reported by us [14]. For example, notice in Fig. 5 how convergence was achieved at 100 epoch iterations reaching accuracies above $94 \%$ for all shapes. In contrast, our previous results, used 1800 iterations and still some shapes such as cubes and cylinders had misclassifications above $25 \%$.

\subsection{An example data input and CAM}

We can represent light-scattering data to display the Mueller matrix in a planar data grid for all angles. For example, Fig. 7 illustrates the same data as that of Fig. 4 for all elements $S_{i j}$ of the Mueller matrix and for all angles from $0^{\circ} \leq \theta \leq 180^{\circ}$ and $0^{\circ} \leq \varphi$ $\leq 360^{\circ}$.

All these elements, when processed by our ML architecture produce CAMs as described in Section 2.3. The resulting CAM is shown in Fig. 8. 


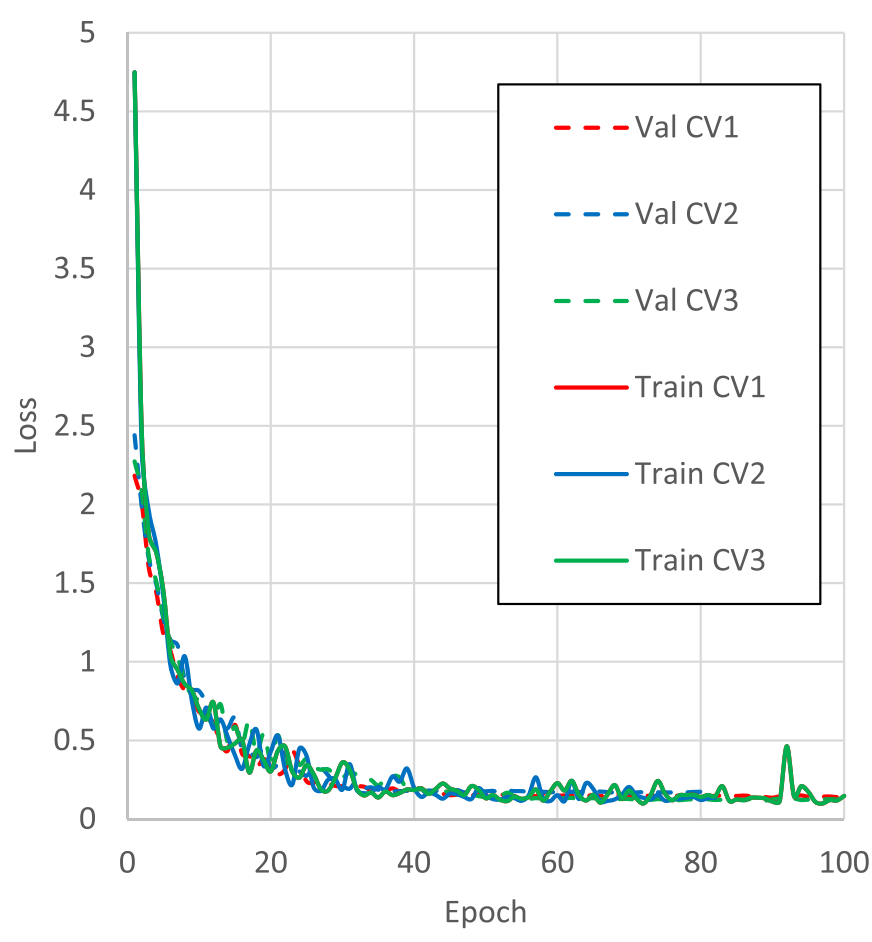

Fig. 6. Plot of loss over each epoch in training for the validation and training subsets during cross-validation experiments.
Fig. 8 shows sample activation regions for one specific particle in a specific orientation. These regions of activation are unique for each particle and orientation. In addition, these markers of activation are determined by the combination of weights and biases that were optimized while training. If these weights are changed, the optimization maps will change. However, we note that we have trained the weights to optimize classification from our training dataset. We do not expect that finding a different local optimization will change this map drastically. The asymptotic limit of the loss function and near maximum classification metrics across the all cross-validation experiments indicates near optimum weights have been learned. The ML architecture evaluates the combination of activation regions and decides which type of particle is the most likely to produce this pattern of activation.

\subsection{Azimuthally averaged CAMS}

To make sense of these CAMs and have some generalizable insights, we calculate CAMs for 2000 samples of each type of particle. Since we also consider particle orientation in the averaging, the dependence on the azimuthal angle vanishes and eventually a constant fringe of activation along the azimuthal angle appears. Since our activation function is ReLU, it is not normalized and the activation yields a uniform evaluation value of how useful or not the region is to perform a classification decision. Hence, in order to compare the activation of different shapes of particles, we normalize the CAMs by division by their maximal values. In this way, we can compare the activation of each region. In other words, we $\log _{10} S_{11}$

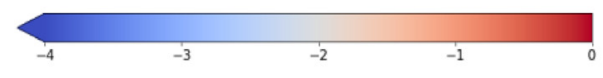

\section{$S_{i j}^{*}$}

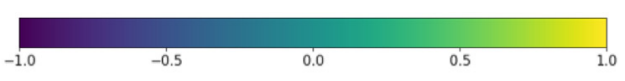

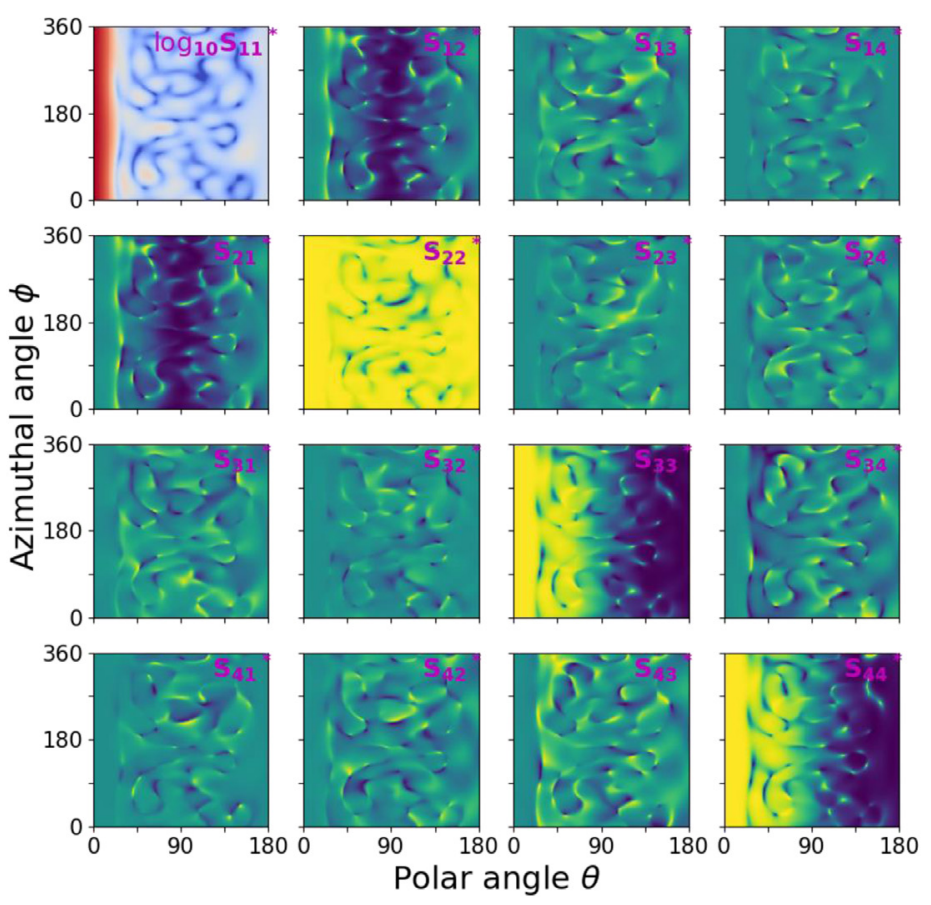

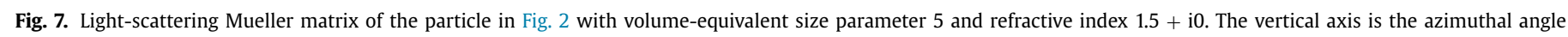
$\varphi$ while the horizontal axis is the polar angle $\theta$. 

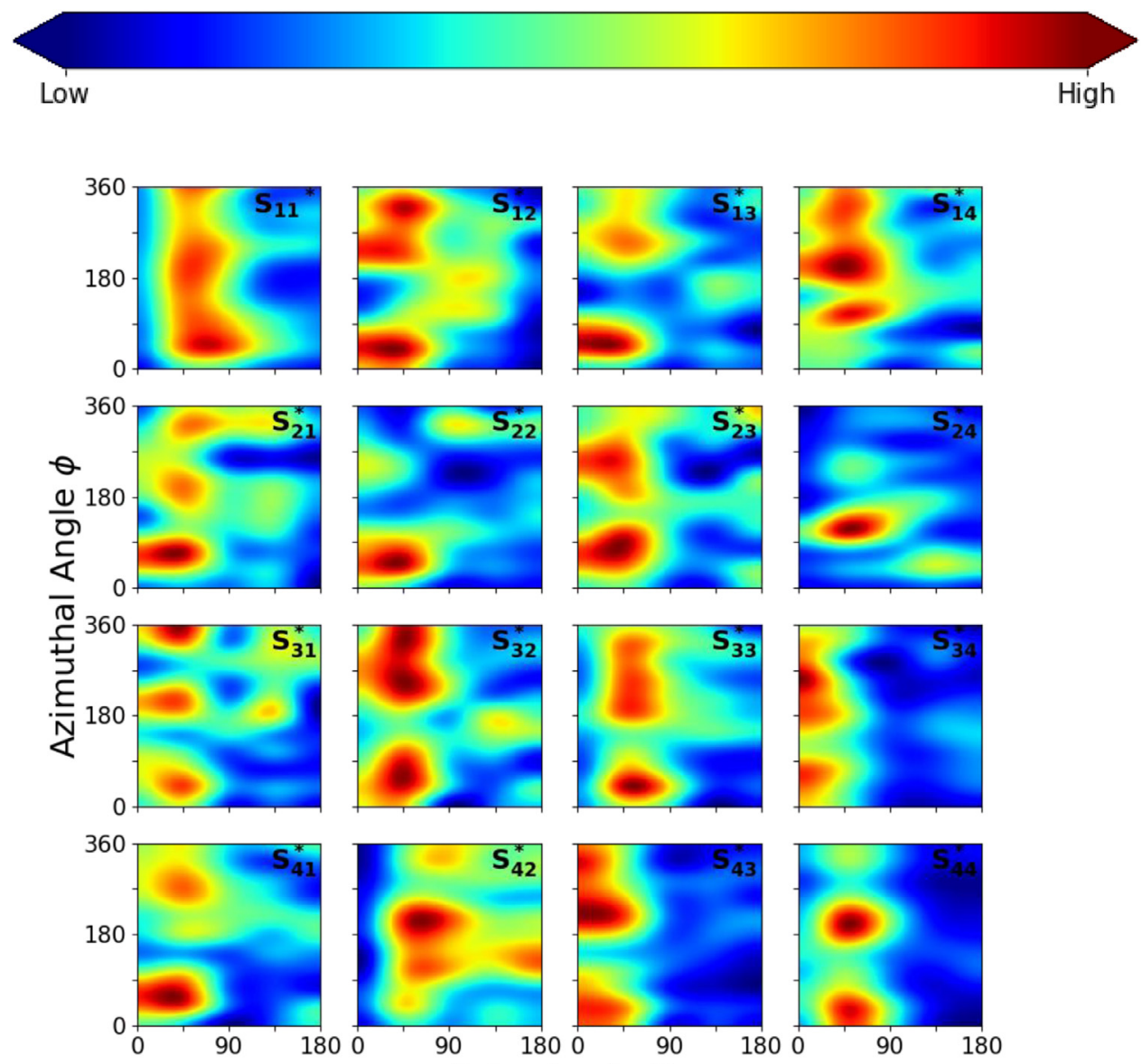

Polar Angle $\theta$

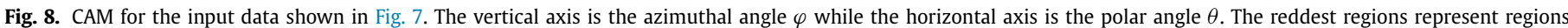

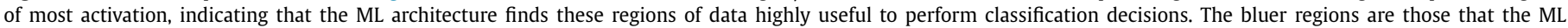
finds less useful for decision making.

show where, along the polar-angle, features occur that ML uses to differentiate one class of particles from another. We show this data in Fig. 9.

As can be seen in Fig. 9, the activation region is different for each type of particle and Mueller matrix element. This means that if our goal is to detect cuboids, we may want to consider setting up detectors in different locations and consider different polarization states than if we are trying to detect agglomerated debris. The peak of activation of $S_{11}^{*}$ is perhaps one of the most intuitive as the region of maximum activation for most particles is located in the near-forward scattering region between $\sim 20^{\circ}$ to $\sim 70^{\circ}$. This range of angular discrimination is often referred to as the diffraction regime, which is known to be highly dependent on particle shape. Indeed, detector systems have taken advantage of this region for decades [32,33] and continues to the present day in aerosol classification [34]. The peak of the CAMs in this region suggests that light-scattering features are distinct in this region. While the use of the diffraction region for particle differentiation is based on physical principles, the ML architecture confirms this through bruteforce analysis. Another interesting observation occurs in the linearpolarization element $S_{12}^{*}$, which peaks at approximately $\sim 30^{\circ}$ for most shapes, except for sphere and spore clusters whose classification activity peaks at $\sim 100^{\circ}$. The scattering angles recognized as the most discriminative for these two particular classes of particles showcase the usefulness of CAMs as a tool for leveraging ML as means of discovering discriminative features among particle types. Spore clusters and sphere clusters were the most similar classes of particles created by Piedra et al. [14]. As such, we considered these two classes would present a difficult discrimination challenge. Hence, we see that the ML optimized its activation and found the diffraction region as the most discriminative; however, for the $S_{12}^{*}$ data from spore and sphere clusters, the ML also found mid-range angles $\sim 100^{\circ}$ as being equally discriminative as the diffractive region. This demonstrates that CAMs can be leveraged as a tool to highlight discriminative light-scattering angles, which might not be inherently intuitive a priori. We expected the fractal aggregates also to have peaked near $\sim 90^{\circ}$, because they tend to be composed of nearly non-interacting dipoles that have a strong TE polarization near $100 \%$ in this region, but it turns out that nearforward region is actually more efficient at classifying such particles given this dataset. This possibly is because such porous particle do not have strong diffraction rings, differentiating them from other particle shapes that we considered. Other interesting elements are $S_{33}^{*}, S_{34}^{*}, S_{43}^{*}$ and $S_{44}^{*}$ that can have a bimodal activation region with a minimum near $90^{\circ}$, especially for the clusters. Other particle shapes may have a fairly flat response. This flat response is seen for other Mueller matrix elements as well. For instance, Mueller matrix element $S_{22}^{*}$ maintains nearly constant activation for all shapes between $30^{\circ} \leq \theta \leq 150^{\circ}$. This indicates that using this element will achieve nearly the same usefulness across this range of polar angles.

In Fig. 10, we present the resulting azimuthally averaged activation map for 1000 particles selected at random. This exploration is analogous to a scenario when a light-scattering discrimination sys- 

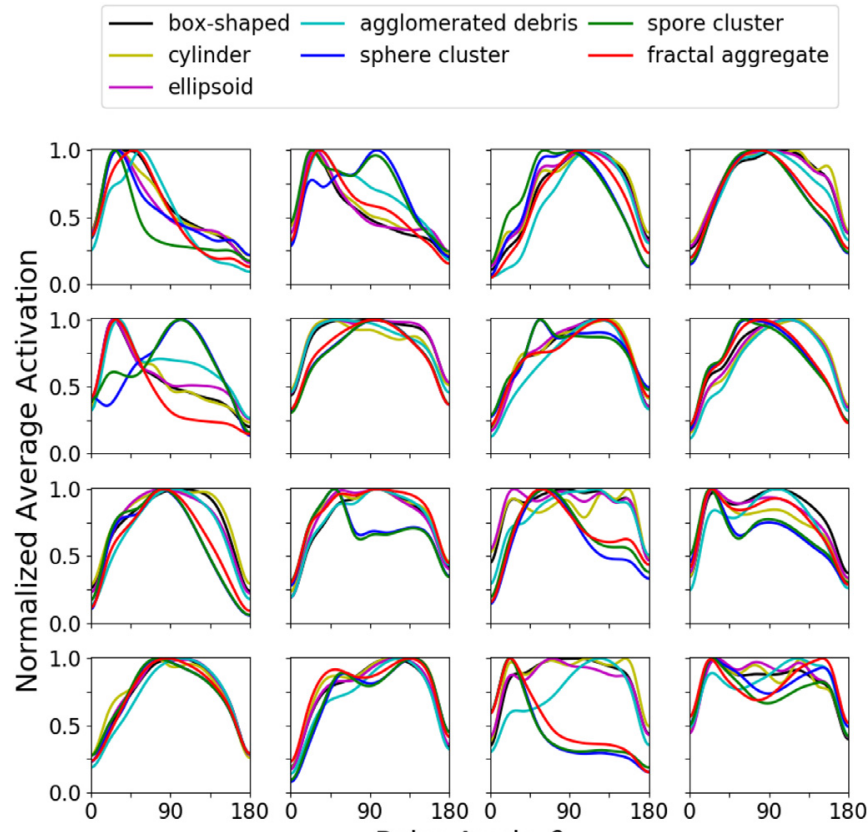

Polar Angle $\theta$

Fig. 9. Average activation maps for 2000 discriminated particles. The order of the matrix of activation curves is the same as that of Fig. 8. The final activation map is nearly azimuthally independent and is normalized with respect to its maximum value. Here we display the normalized, azimuthally averaged activation performance of light-scattering data as function of polar angle.

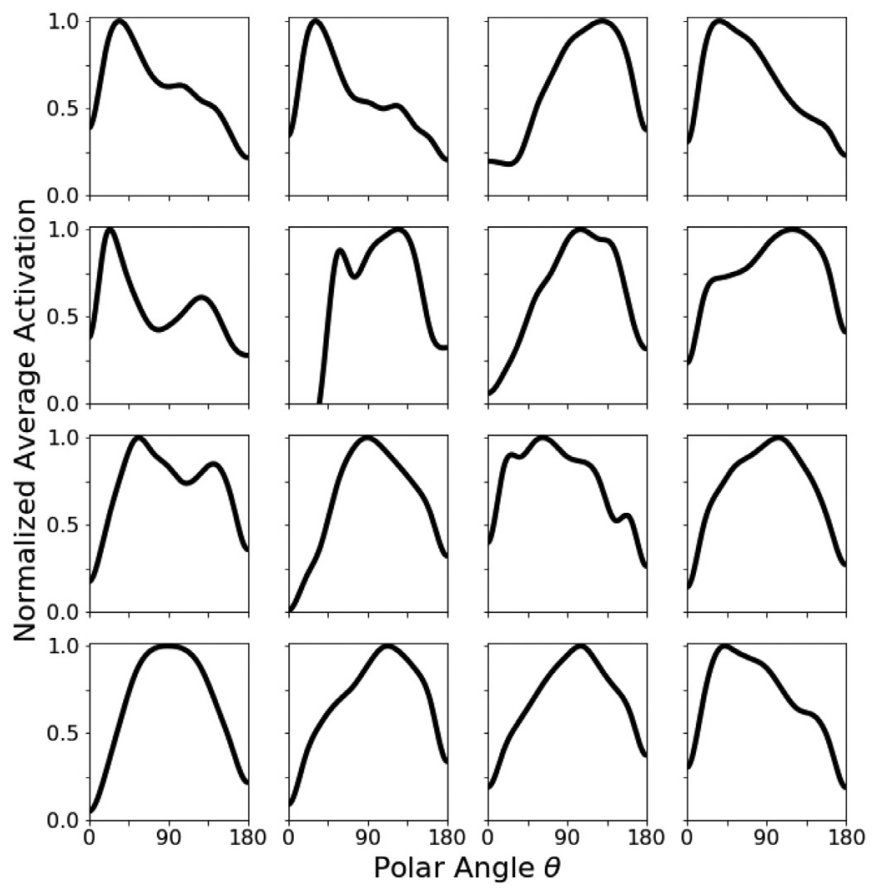

Fig. 10. Normalized CAM for 1000 particles selected at random. The final activation map is shown as a function of polar angle since the overall activation map is independent of the azimuthal component for a large number of particles.

tem does not know what types of particles are being differentiated a prori. The CAMs highlight which scattering angles are most discriminative for all particles in common. We have normalized this curve with respect to the highest activation of the combined set of particles. As in Fig. 9, the resulting CAM is a function of the polar scattering angle $\theta$ only. These activation maps present some interesting results. For instance, for the $S_{11}^{*}, S_{14}^{*} \quad S_{12}^{*}$ and $S_{21}^{*}$ training datasets, the ML architecture found the region about the polar angle $\sim 35^{\circ}$ to be most useful for classification while still maintaining a fairly large activitation greater than 0.5 out to $\sim 120^{\circ}$. For the element $S_{12}^{*}$, the architecture found the angle $\sim 30^{\circ}$ to be most useful, and largely found the backscattering from $\theta \approx 125^{\circ}$ to be the slightly less useful for elements $S_{12}^{*}$ and $S_{21}^{*}$ than for element $S_{11}^{*}$. In general, the other polarization matrix elements did not find that forward-scatter region to be as strong a classifier as for these elements. As expected from the results of Fig. 9, the diffraction region of the total intensity element $S_{11}^{*}$ provides strong overall classification differentiation for all particle shapes.

Some datasets such as $S_{13}^{*}, S_{22}^{*}, S_{24}^{*}, S_{34}^{*}, S_{42}^{*}$, and $S_{43}^{*}$ have activations that peak in the backscattering hemisphere between $110^{\circ} \lesssim$ $\theta \lesssim 130^{\circ}$. In the far backscattering region where scattering angle $\theta$ approaches $180^{\circ}$, we do not see significant activation of any of the Mueller matrix elements. Coherent effects play a dominant role in this scattering regime, which are manifest in a backscattering surge and accompanying negative polarization branch [35,36]. The masking simulations of Bickel et al. [37] suggest that this region is especially sensitive to small changes in particle morphology. It appears that this region is too sensitive to be used as a classifier of different particle classes as defined in this particular experiment, but may be useful to provide information about particle subclasses, like the size of particles making up an agglomerate as suggested by Zubko et al. [38].

\section{Conclusions}

In this work, we release our ML code, ScatCAM, which can be accessed at https://github.com/USArmyResearchLab/ScatCAM/. We show how ML techniques can be used to locate regions of light-scattering patterns that possess discriminating features. We demonstrate a method for mitigating the poor understanding of ML methods, i.e., the ML black-box problem. We investigate how a ML architecture learns to classify particles using the scattering dataset that was studied previously [14]. CNN-architecture hyperparameter tuning and choices including leaky ReLU, batch normalization, and residual connections allow for significant improvements in accuracy and convergence speed in comparison with our previous results. This study explores the CAM technique by modifying our $\mathrm{CNN}$ to include a global-average pooling layer. By doing so, we are able to obtain a weighted map such as Fig. 8 that highlights the most unambiguous features of the input light-scattering dataset, provided that the ML optimized its weights and biases during training. We calculate how each Mueller matrix element is activated as a function of polar angle $\theta$ and azimuthal angle $\varphi$. We also produce average maps of this function over many such particles of the same class. For such populations, the azimuthal dependence disappears and we only plot the polar-angle dependence. This quantity is a measure of how useful the ML finds the data for classification decisions in comparison to the most useful region. Overall, we find that class-activation mapping recognizes the forward-scattering, diffraction region to be most discriminative for analyzing the total intensity signals. Interestingly, for other polarization elements, the most useful classification regions depend on particle shape (or class). For example, for two classes of particles with extremely similar light-scattering profile, our CAMs were able to highlight discriminative scattering angles at $\sim 100^{\circ}$. This angle might not necessarily be an intuitive choice to perform a particle-discrimination experiment. These result highlight that ML can potentially be used for guidance as to which scattering angles can present the strongest classification profiles. Such a tool can be used in different applications, for instance in determining where to place detectors to classify particles most efficiently. Such applications range from flow cytometry to characterize blood cells to satellite remote sensing to determine atmospheric components. 


\section{Declaration of Competing Interest}

None.

\section{CRediT authorship contribution statement}

Patricio Piedra: Conceptualization, Methodology, Software, Validation, Data curation, Investigation, Writing - original draft, Writing - review \& editing, Visualization, Supervision, Project administration, Funding acquisition. Christian Gobert: Methodology, Software, Formal analysis, Investigation, Data curation, Writing original draft, Writing - review \& editing, Visualization. Aimable Kalume: Conceptualization, Methodology, Writing - original draft, Writing - review \& editing. Yong-Le Pan: Conceptualization, Writing - original draft, Writing - review \& editing, Resources, Supervision, Funding acquisition. Miroslav Kocifaj: Validation, Writing - original draft, Writing - review \& editing. Karri Muinonen: Validation, Writing - original draft, Writing - review \& editing. Antti Penttilä: Validation, Writing - original draft, Writing - review \& editing. Evgenij Zubko: Conceptualization, Software, Validation, Writing - original draft, Writing - review \& editing. Gorden Videen: Conceptualization, Methodology, Validation, Investigation, Resources, Writing - original draft, Writing - review \& editing, Supervision, Funding acquisition.

\section{Acknowledgements}

Patricio Piedra kindly acknowledges the support of this work by the Defense Threat Reduction Agency (DTRA) under the Research Associate Program of the National Research Council and by sponsorship from the U.S. Army Research Laboratory. The views and conclusions contained in this document are those of the authors and should not be interpreted as representing the official policies, either expressed or implied, of the Combat Capabilities Development Command of the Army Research Laboratory or the U.S. Government. The U.S. Government is authorized to reproduce and distribute reprints for Government purposes notwithstanding any copyright notation herein. Miroslav Kocifaj was supported by the Slovak Research and Development Agency under Project No. APVV18-0014.

\section{Supplementary materials}

Supplementary material associated with this article can be found, in the online version, at doi:10.1016/j.jqsrt.2020.106936.

\section{References}

[1] Pan Y-L, Aptowicz KB, Chang RK, Hart M, Eversole JD. Characterizing and monitoring respiratory aerosols by light scattering. Opt Lett 2003;28:589-91.

[2] Jarvis RM, Brooker A, Goodacre R. Surface-enhanced Raman scattering for the rapid discrimination of bacteria. Faraday Discuss 2006;132:281-92.

[3] Seaver M, Eversole JD, Hardgrove JJ, Cary WK, Roselle DC. Size and fluorescence measurements for field detection of biological aerosols. Aero Sci Technol 1999;30:174-85.

[4] Pan Y-L, Hill SC, Pinnick RG, Huang H, Bottiger JR, Chang RK. Fluorescence spectra of atmospheric aerosol particles measured using one or two excitation wavelengths: comparison of classification schemes employing different emission and scattering results. Opt Express 2010;18:12436-57.

[5] Holler S, Zomer S, Crosta GF, Chang RK, Bottiger JR. Multivariate analysis and classification of two-dimensional angular optical scattering patterns from aggregates. Appl Opt 2004;43:6198-206.

[6] Aptowicz K, Pinnick R, Hill S, Pan Y, Chang R. Optical scattering patterns from single urban aerosol particles at Adelphi, Maryland, USA: a classification relating to particle morphologies. J Geophys Res 2006;111.

[7] Crosta GF, Pan Y-L, Aptowicz KB, Casati C, Pinnick RG, Chang RK, et al. Automated classification of single airborne particles from two-dimensional angle-resolved optical scattering (TAOS) patterns by non-linear filtering. J Quantit Spectrosc Radiat Transf 2013;131:215-33.

[8] Aptowicz KB, Pan Y-L, Martin SD, Fernandez E, Chang RK, Pinnick RG. Decomposition of atmospheric aerosol phase function by particle size and asphericity from measurements of single particle optical scattering patterns. J Quantit Spectrosc Radiat Transf 2013;131:13-23.
[9] Genuer V, Gal O, Méteau J, Marcoux P, Schultz E, Lacot É, et al. Optical elastic scattering for early label-free identification of clinical pathogens. Advanced Biomedical and Clinical Diagnostic and Surgical Guidance Systems, XIV. In: Proceedings of International Society for Optics and Photonics, 969896980A; 2016.

[10] Sivaprakasam V, Lin H-B, Huston AL, Eversole JD. Spectral characterization of biological aerosol particles using two-wavelength excited laser-induced fluorescence and elastic scattering measurements. Opt. Express 2011;19:6191-208.

[11] Bishop CM. Pattern recognition and machine learning. Springer; 2006.

[12] Kaye P, Hirst E, Wang-Thomas Z. Neural-network-based spatial light-scattering instrument for hazardous airborne fiber detection. Appl Opt 1997;36:6149-56.

[13] Kuo CCJ, Zhang M, Li S, Duan J, Chen Y. Interpretable convolutiona neural networks via feedforward design. J Vis Commun Image Represent 2019;60:346-59.

[14] Piedra P, Kalume A, Zubko E, Mackowski D, Pan Y-L, Videen G. Particle-shape classification using light scattering: an exercise in deep learning. J Quantit Spectrosc Radiat Transf 2019;231:140-56.

[15] Lin M., Chen Q., Yan S. Network in network 2013; arXiv preprint arXiv:1312. 4400 .

[16] Zhou B, Khosla A, Lapedriza A, Oliva A, Torralba A. Learning deep features for discriminative localization. In: Proceedings of the IEEE conference on computer vision and pattern recognition; 2016. p. 2921-9.

[17] Ulanowski Z, Hirst E, Kaye PH, Greenaway R. Retrieving the size of particles with rough and complex surfaces from two-dimensional scattering patterns. J Quantit Spectrosc Radiat Transf 2012;113:2457-64.

[18] Brunel M, Shen H, Coëtmellec S, Gréhan G, Delobel T. Determination of the size of irregular particles using interferometric out-of-focus imaging. Int J Opt $2014 ; 2014$.

[19] LeCun Y, Kavukcuoglu K, Farabet C. Convolutional networks and applications in vision. In: Proceedings of 2010 IEEE International Symposium on Circuits and Systems: IEEE; 2010. p. 253-6.

[20] LeCun Y, Bengio Y, Hinton G. Deep learning. Nature 2015;521:436

[21] Krizhevsky A, Sutskever I, Hinton GE. Imagenet classification with deep convolutional neural networks. Adv Neur Inform Process Syst 2012:1097-105.

[22] Chollet F., others. Keras. https://keras.io; 2015.

[23] Nwankpa, C., Ijomah, W., Gachagan, A., Marshall, S. Activation functions: Comparison of trends in practice and research for deep learning. arXiv 2018; arXiv preprint arXiv: 1811.03378

[24] Ioffe S, Szegedy C. Batch normalization: accelerating deep network training by reducing internal covariate shift. In: Proceedings of the 32 nd International Conference on International Conference on Machine Learning (ICML'15), 37; 2015. p. 448-56. JMLR.org.

[25] He K, Zhang X, Ren S, Sun J. Deep residual learning for image recognition. In: Proceedings of the IEEE conference on computer vision and pattern recognition; 2016. p. 770-8.

[26] Ng AY. Feature selection, L 1vs. L 2 regularization, and rotational invariance. In: Proceedings of the twenty-first international conference on machine learning: ACM; 2004. p. 78.

[27] Ruder S. An overview of gradient descent optimization algorithms. arXiv preprint 2016.

[28] Mishchenko MI, Hovenier JW, Travis LD. Light scattering by nonspherical particles: theory, measurements, and applications. IOP Publishing; 2000.

[29] Piedra PG, Moosmüller H. Optical losses of photovoltaic cells due to aerosol deposition: role of particle refractive index and size. Solar Energy 2017; 155:637-46.

[30] Hulst HC, van de Hulst HC. Light scattering by small particles. Courier Corporation; 1981.

[31] Fawcett T. An introduction to ROC analysis. Pattern Recognit Lett 2006;27:861-74.

[32] Hirst E, Kaye PH, Guppy JR. Light scattering from nonspherical airborne particles: experimental and theoretical comparisons. Appl Opt 1994;33:7180-6.

[33] Kaye PH, Alexander-Buckley K, Hirst E, Saunders S, Clark JM. A real-time monitoring system for airborne particle shape and size analysis. J Geophys Res 1996;101:19215-21.

[34] Haddrell A, Rovelli G, Lewis D, Church T, Reid J. Identifying time-dependent changes in the morphology of an individual aerosol particle from their light scattering patterns. Aero Sci Technol 2019:1-18.

[35] Muinonen K, Tyynelä J, Zubko E, Lindqvist H, Penttilä A, Videen G. Polarization of light backscattered by small particles. J Quantit Spectrosc Radiat Transf 2011;112:2193-212.

[36] Videen G., Kocifaj M. Optics of cosmic dust: Springer Science \& Business Media; 2002.

[37] Bickel WS, Yousif HA, Bailey WM. Masking of information in light scattering signals from complex scatterers. Aero Sci Technol 1982;1:329-35.

[38] Zubko E, Shkuratov Y, Hart M, Eversole J, Videen G. Backscatter of agglomerate particles. J Quantit Spectrosc Radiat Transf 2004;88:163-71.

[39] Haddrell A, Rovelli G, Lewis D, Church T, Reid J. Identifying time-dependent changes in the morphology of an individual aerosol particle from its light scattering pattern. Aerosol Science and Technology 2019;53(11):1334-51. doi:10 1080/02786826.2019.1661351.

[40] Hamill P, Piedra P, Giordano M. Simulated polarization as a signature of aerosol type. Atmos. Environ. 2020;224(117348). doi:10.1016/j.atmosenv.2020.117348. 